\title{
Whole-genome sequence assembly of Pediococcus pentosaceus LI05 (CGMCC 7049) from the human gastrointestinal tract and comparative analysis with representative sequences from three food-borne strains
}

\author{
Long-Xian Lv $v^{1,3}$, Yu-Dong Li $i^{2}$, Xin-Jun Hu ${ }^{1,3}$, Hai-Yan Shi $i^{1,3}$ and Lan-Juan Li $i^{1,3^{*}}$
}

\begin{abstract}
Background: Strains of Pediococcus pentosaceus from food and the human gastrointestinal tract have been widely identified, and some have been reported to reduce inflammation, encephalopathy, obesity and fatty liver in animals. In this study, we sequenced the whole genome of P. pentosaceus LI05 (CGMCC 7049), which was isolated from the fecal samples of healthy volunteers, and determined its ability to reduce acute liver injury. No other genomic information for gut-borne $P$. pentosaceus is currently available in the public domain.

Results: We obtained the draft genome of $P$. pentosaceus LI05, which was 1,751,578 bp in size and possessed a mean $\mathrm{G}+\mathrm{C}$ content of $37.3 \%$. This genome encoded an abundance of proteins that were protective against acids, bile salts, heat, oxidative stresses, enterocin A, arsenate and universal stresses. Important adhesion proteins were also encoded by the genome. Additionally, P. pentosaceus LI05 genes encoded proteins associated with the biosynthesis of not only three antimicrobials, including prebacteriocin, lysin and colicin $\mathrm{V}$, but also vitamins and functional amino acids, such as riboflavin, folate, biotin, thiamine and gamma-aminobutyrate. A comparison of $P$. pentosaceus LI05 with all known genomes of food-borne $P$. pentosaceus strains (ATCC 25745, SL4 and IE-3) revealed that it possessed four novel exopolysaccharide biosynthesis proteins, additional putative environmental stress tolerance proteins and phage-related proteins.

Conclusions: This work demonstrated the probiotic properties of P. pentosaceus LI05 from the gut and the three other food-borne $P$. pentosaceus strains through genomic analyses. We have revealed the major genomic differences between these strains, providing a framework for understanding the probiotic effects of strain LI05, which exhibits unique physiological and metabolic properties.
\end{abstract}

\section{Background}

The genus Pediococcus belongs to the family Lactobacillaceae in the order Lactobacillales. Currently, it is comprised of eleven valid published species, including Pediococcus acidilactici, P. stilesii, P. pentosaceus, P. siamensis, P. cellicola, $P$. argentinicus, $P$. parvulus, $P$. ethanolidurans,

\footnotetext{
* Correspondence: lji@@ju.edu.cn

'State Key Laboratory for Diagnosis and Treatment of Infectious Disease, The First Affiliated Hospital, Zhejiang University, Hangzhou 310003, PR China ${ }^{3}$ Collaborative Innovation Center for Diagnosis and Treatment of Infectious Diseases, Hangzhou, China

Full list of author information is available at the end of the article
}

P. claussenii, P. inopinatus and P. damnosus [1]. The majority of the members of the genus Pediococcus are used in the food and drink industry as starter and probiotic cultures as well as food spoilers [2]. P. pentosaceus has been intensively investigated and widely employed for food preservation due to its ability to produce antimicrobial agents [3]. Additionally, several strains of $P$. pentosaceus have been shown to reduce inflammation, encephalopathy [4], obesity and fatty liver [5] in animals. Although food is the main source of $P$. pentosaceus for humans, the strains of $P$. pentosaceus adapted to the gastrointestinal tract are dissimilar from those found 
in food because the former may originate from subpopulations present in food at low numbers that exhibit special adaptive properties [6].

Previously, we have isolated a potential probiotic, P. pentosaceus LI05 (CGMCC 7049), from the fecal samples of healthy volunteers. This strain is tolerant to bile and acid and possesses strong antimicrobial activities against tested enteropathogens. More importantly, the administration of $P$. pentosaceus LI05 during acute D-galactosamine-induced liver injury in rats was shown to reduce elevated alanine aminotransferase and aspartate aminotransferase levels, prevent the increase of total bilirubin, reduce the histological abnormalities of both the liver and terminal ileum, decrease bacterial translocation, increase the serum levels of IL-10 and result in a cecal microbiome that differ from that of the liver injury control [7].

In this study, we present a summary, classification and the unique characteristics of human gut-borne $P$. pentosaceus LI05 in addition to a high-quality draft genome sequence and annotations. The probiotic properties of $P$. pentosaceus LI05 were analyzed using these genomic sequences combined with data from our previous study. Because the genome sequences of $P$. pentosaceus SL4 from kimchi [8], P. pentosaceus IE-3 from a dairy effluent sample [9], and $P$. pentosaceus ATCC25745 from plant [10] are now available, this research will provide an essential resource for elucidating the differences between strains isolated from food and the human gastrointestinal tract.

\section{Methods}

Determination of cultural, morphological and physiological properties

Growth was investigated under different temperature, $\mathrm{pH}$ and $\mathrm{NaCl}$ conditions. Cell morphologies, motilities and sporulation activities were examined using transmission electron (H-600, Hitachi Ltd., Tokyo, Japan) microscopy. Phenotypic identification was achieved with API CH50 strips and the API CHL medium system according to the manufacturer's instructions (BioMérieux SA, Marcy-l'Etoile, France). Other physiological and biochemical tests were conducted as described previously [11]. Phylogenetic analysis was conducted using the neighbor-joining method based on the 16S rRNA and housekeeping gene sequences [12].

\section{Cultural conditions and DNA isolation}

After revival using standard methods, the $P$. pentosaceus LI05 strain (CGMCC 7049) was anaerobically cultured in DeMan-Rogosa-Sharpe (MRS; OXOID, Thermo Fisher Biochemicals Ltd., Beijing, China) broth at $37^{\circ} \mathrm{C}$ for $24 \mathrm{~h}$. Cells were obtained by centrifugation at $8,000 \mathrm{~g}$ for $10 \mathrm{~min}$ at $4^{\circ} \mathrm{C}$. DNA was extracted using the QIAamp
DNA Micro Kit according to manufacturer's guidelines (Qiagen, Westburgb.v., Leusden, The Netherlands).

\section{Genome sequencing and assembly}

The genome of $P$. pentosaceus LI05 was sequenced with the next-generation sequencing platform Illumina HiSeq 2000 , and the total number of reads based on a 500-bp library database were $2 \times 11,079,017$ (bp). The quality of the sequencing read data was estimated by calculating the quality and GC content of each read. The draft genome sequence was assembled using SOAPdenovo2 [13], and iterative optimization was used to obtain the optimal k-mer value through the use of 31-85 k-mers. The 500-bp libraries were used to build scaffolds, and the SOAPdenovo gap closer software was also used (http:// soap.genomics.org.cn/soapdenovo.html). To close the remaining gaps, reference-guided assemblies were carried out with the CLC Genomics Workbench v. 6.05 (CLC bio, Aarbus, Denmark). The combination of de novo assembly and reference-guided assembly was performed manually using the microbial genome-finishing module in the CLC genomics workbench (CLC bio, Aarbus, Denmark). The complete genome sequence of $P$. pentosaceus ATCC 25745 was used as the reference genome.

\section{Genome annotation}

$P$. pentosaceus LI05 genes were identified using Glimmer [14] together with comparative gene prediction by the direct mapping of the ORFs of the P. pentosaceus ATCC reference strain from the NCBI Genome Database. After a round of manual curation, the unannotated predicted coding sequences (CDS) were translated into amino acid sequences for a query using the NCBI non-redundant database as well as the UniProt, Pfam, COG, and InterPro databases to identify the closest existing homology annotations. Transfer RNA (tRNA) genes were detected using tRNAScanSE [15]. Ribosomal RNAs (rRNAs) were identified using a BLASTn [16] search against the ribosomal RNA databases. Signal peptides were predicted using SignalP 4.0 [17], whereas transmembrane helices in proteins were predicted using TMHMM [18]. The Integrated Microbial Genomes (IMG) platform (http://img.jgi. doe.gov/) was used to support additional gene prediction analyses and manual functional annotations [19].

\section{Comparative genomics}

A comparative genomic analysis using BRIG [20] was conducted comparing P. pentosaceus LI05 from the human gastrointestinal tract with three food-borne strains with available genomic sequences, including $P$. pentosaceus ATCC 25745, SL4 and IE-3. The P. pentosaceus LI05 genome sequences sharing low identities $(<50 \%)$ with the other strains were designated as the $P$. pentosaceus LI05-unique regions. The proteins encoded by the 
genes that only existed in $P$. pentosaceus LI05 or that possessed sequence similarities of less than $50 \%$ with the three food-borne strains were further analyzed by BLASTp.

\section{Results and discussion} Classification and unique features

P. pentosaceus LI05 is a Gram-negative, non-motile, acid-tolerant, non-sporulating, spherical, facultative anaerobe from the human gastrointestinal tract (Additional file 1: Figure S1). It tolerates $6 \% \mathrm{NaCl}$ in $\mathrm{MRS}$ broth. Growth occurs at $15-45^{\circ} \mathrm{C}$ and at $\mathrm{pH} 4-8$ but optimally at $37^{\circ} \mathrm{C}$. The colonies on the MRS agar were white, smooth, shiny, and circular with complete edges. Some carbohydrates, such as L-arabinose, D-ribose, D-xylose, $\mathrm{D}$-galactose, D-glucose, D-fructose, D-mannose, Nacetylglucosamine, amygdalin, arbutin, salicin, D-cellobiose, D-maltose, D-trehalose, gentiobiose, and D-fucose, can be used as the sole carbon sources, whereas glycerol, erythritol, etc. cannot (Additional file 2: Table S1).

A neighbor-joining tree (Figure 1A) based on the $16 \mathrm{~S}$ rRNA gene sequence of the strain LI05 shows the phylogenetic relationships between the species of the genus Pediococcus. This organism formed a distinct branch with $P$. pentosaceus, which was separate from those formed by other members of the genus Pediococcus. Sequence analyses of the dnaA, dnaJ, dnaK, pheS, pryH, recA, recH, tuF, gry $B$ and $r p l B$ housekeeping genes were carried out for the definitive identifications of $P$. pentosaceus LI05, P. pentosaceus ATCC 25745, P. pentosaceus SL4 and P. pentosaceus IE-3. As shown in Figure 1B, the combination of the above housekeeping genes provided good phylogenetic resolution of the four strains. The $P$. pentosaceus strain IE-3 was the closest evolutionary relative of strain LI05.

\section{Genome properties}

The genome of $P$. pentosaceus LI05 was sequenced by the Illumina method (see Methods). A total of 11.05 million 100-bp paired-end reads were generated, which provided over 500-fold coverage of the reference genome. High-quality reads with $\mathrm{Q}>30$ were assembled using de novo methods to obtain a draft genome of $1.75 \mathrm{Mbp}$ with 8 contigs (the $\mathrm{N} 50$ of the assembled contigs was $34.3 \mathrm{~Kb}$; the max length was $318 \mathrm{~Kb}$ ). The $\mathrm{G}+\mathrm{C}$ content of $P$. pentosaceus LI05 was $37.29 \%$. For the main chromosome, 1,638 genes were predicted, 1,555 of which were protein-coding genes. A total of 1,321 proteincoding genes were assigned to putative functions, and the remainder were classified as hypothetical proteins. This genome contained 50 tRNAs and a complete 5S23S-16S rRNA gene family. The properties and statistics of the genome are shown in Table 1 and Figure 2. As shown in Figure 3, the genome sequence of $P$. pentosaceus LI05 was highly conserved compared with those of
P. pentosaceus ATCC 25745 , P. pentosaceus SL4 and P. pentosaceus IE-3.

\section{Genome of $P$. pentosaceus LI05 exhibits probiotic properties}

In a previous study, we have observed that $P$. pentosaceus LI05 is resistant to gastric acidity and bile compounds [7]. This was supported by the genomic data from this study, in which a gene encoding cholylglycine hydrolase, which is related to bile salt resistance, and genes encoding F0F1 ATP synthases, which are important for acid tolerance, were detected (Table 2). Additionally, six universal stress proteins (UspA), the chaperone protein DnaJ, the cofactor GrpE, which participates in the hyperosmotic and heat shock responses, the chaperone GroEL, which protects against environmental stresses, an enterocin A immunity family protein, an arsenate reductase, and methionine sulfoxide reductase A, which protects against oxidative stresses, were annotated. These representative stress resistance genes were highly conserved between $P$. pentosaceus LI05 and P. pentosaceus ATCC 25745, P. pentosaceus IE-3 or P. pentosaceus sL4, but most of them showed significant divergences from other species.

The ability to adhere to gastrointestinal mucosa is an important property of most probiotics [21,22]. Several proteins encoded by $P$. pentosaceus LI05 genes had predicted adhesive potentials (Table 3). For example, sortase attaches surface proteins, including enzymes, pilins and adhesion-mediating large surface glycoproteins, to cell walls. Other proteins included a pilin-like competence protein ComGC, elongation factor $\mathrm{Tu}$ (EF-Tu), an enolase capable of binding to host extracellular fibronectin and the pilus biosynthesis protein HicB. Abundant adhesion proteins encoded by the genomic regions were consistent with the strong adhesion properties of $P$. pentosaceus LI05. However, these proteins have also been predicted in the other tested strains. These findings may represent a possible reason for the extensive colonization of $P$. pentosaceus in the gut. However, the examinations of many more genes or proteins may be required to evaluate the adhesive abilities of probiotics.

The $P$. pentosaceus LI05 genes also encoded three antimicrobials, which is consistent with the excellent antimicrobial ability of this strain. As shown in Table 3, genes encoding prebacteriocin were annotated in the genomes of both $P$. pentosaceus LI05 and P. pentosaceus ATCC 25745. Alternatively, the pedA gene (PCPN_1274) encoding pediocin PA-1 was detected in $P$. pentosaceus IE-3, but it was not identical to the prebacteriocin gene of $P$. pentosaceus LI05. Furthermore, genes encoding colicin V, which is a peptide antibiotic that kills sensitive cells by disrupting their membrane potentials [23], were found in these four P. pentosaceus strains. However, the 


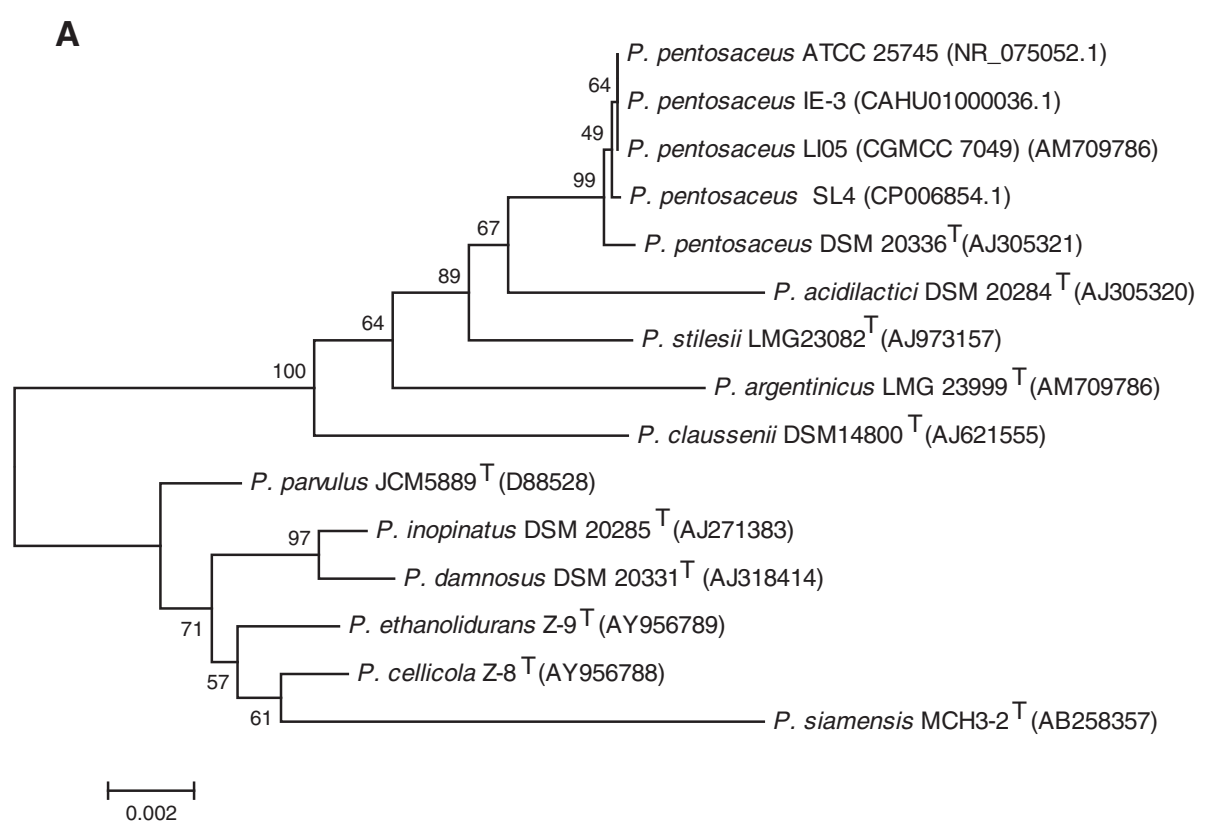

B

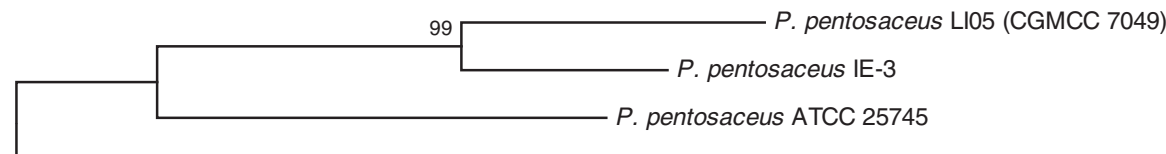

P. pentosaceus SL4

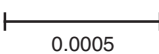

Figure 1 The position of $P$. pentosaceus LI05 relative to the representative strains and the evolutionary relationships of the four strains of $P$. pentosaceus. A. Phylogenetic tree highlighting the position of $P$. pentosaceus $L 105$ relative to the representative strains. The tree was constructed by the neighbor-joining method based on alignments of 165 rRNA gene sequences. Corresponding NCBI accession numbers are shown in parentheses. Numbers at the nodes indicate support values obtained from 1,000 bootstrap replications. B. Phylogenetic tree highlighting the evolutionary relationships of the four strains of $P$. pentosaceus based on concatenated nucleotide sequences of the dnaA, dnaJ, dnaK, pheS, pryH, recA, recH, tuF, gryB and rplB genes.

Table 1 Genomic nucleotide content and gene counts

\begin{tabular}{lll}
\hline Attribute & \multicolumn{2}{l}{ Genome (total) } \\
\cline { 2 - 3 } & Value & \% of total \\
\hline Size (bp) & $1,751,578$ \\
G + C content (bp) & 653,105 & 37.29 \\
Coding region (bp) & $1,457,159$ & 83.19 \\
Total genes & 1,638 & \\
RNA genes & 53 & 3.24 \\
Protein-coding genes & 1,555 & 94.93 \\
Genes assigned to COGs & 1,321 & 84.95 \\
Genes with signal peptides & 29 & 1.86 \\
Genes with transmembrane helices & 492 & 31.64 \\
\hline
\end{tabular}

a) The total is based either on the size of the genome in base pairs or the total number of protein-coding genes in the annotated genome.

${ }^{b)}$ Also includes 35 other genes. colicin V discovered in strain L105 was different from that of the other spicies. Additionally, genes encoding lysin were detected in P. pentosaceus LI05 and P. pentosaceus ATCC 25745. As an antimicrobial agent, lysin is potentially immunogenic [24]. Therefore, $P$. pentosaceus LI05 can achieve "competitive exclusion" not only by limiting the surface area available but also by secreting antimicrobial substances.

In the genome of $P$. pentosaceus LI05, we also detected potentially beneficial properties that were not experimentally confirmed. This strain contained genes involved in the biosynthesis of not only important vitamins, such as riboflavin, folate, thiamine and biotin but also of functional factors, such as gamma-aminobutyrate (Table 3) [25]. In Gram-positive bacteria, peptidoglycan is one of the most important host immune regulators [26]. Although 


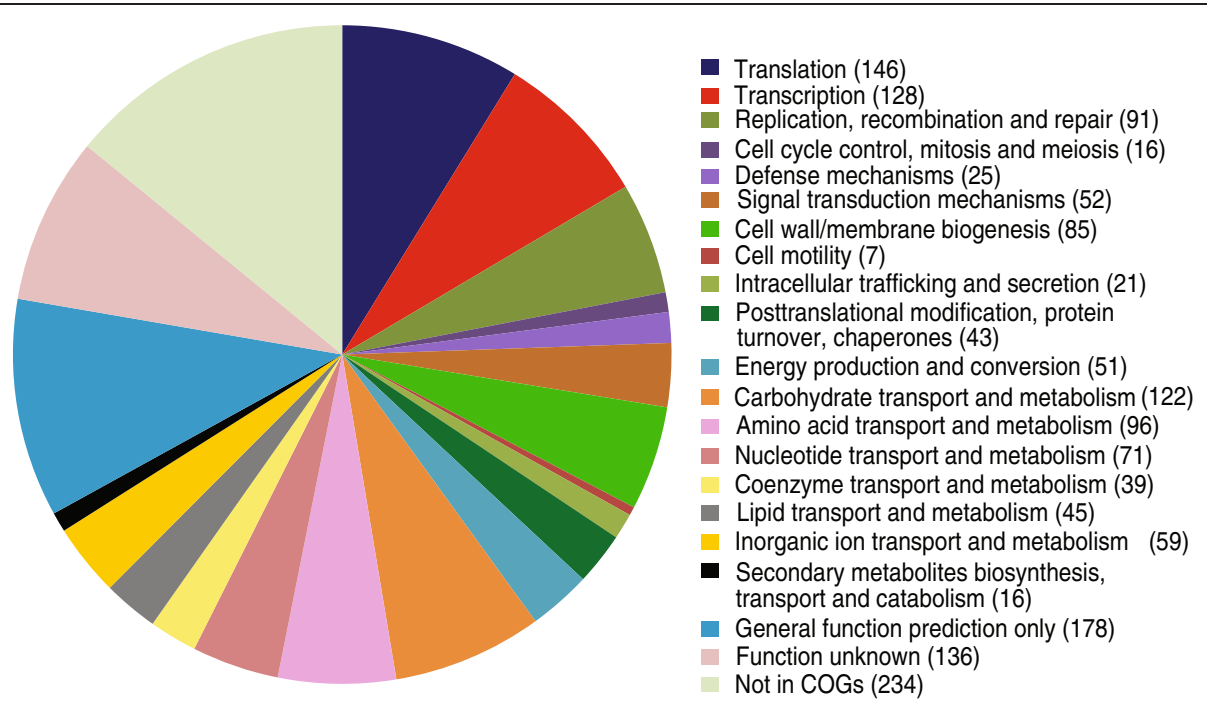

Figure 2 The distribution of the genes associated with the $\mathbf{2 5}$ general COG functional categories in P. pentosaceus LI05. The number of genes is shown in parentheses.

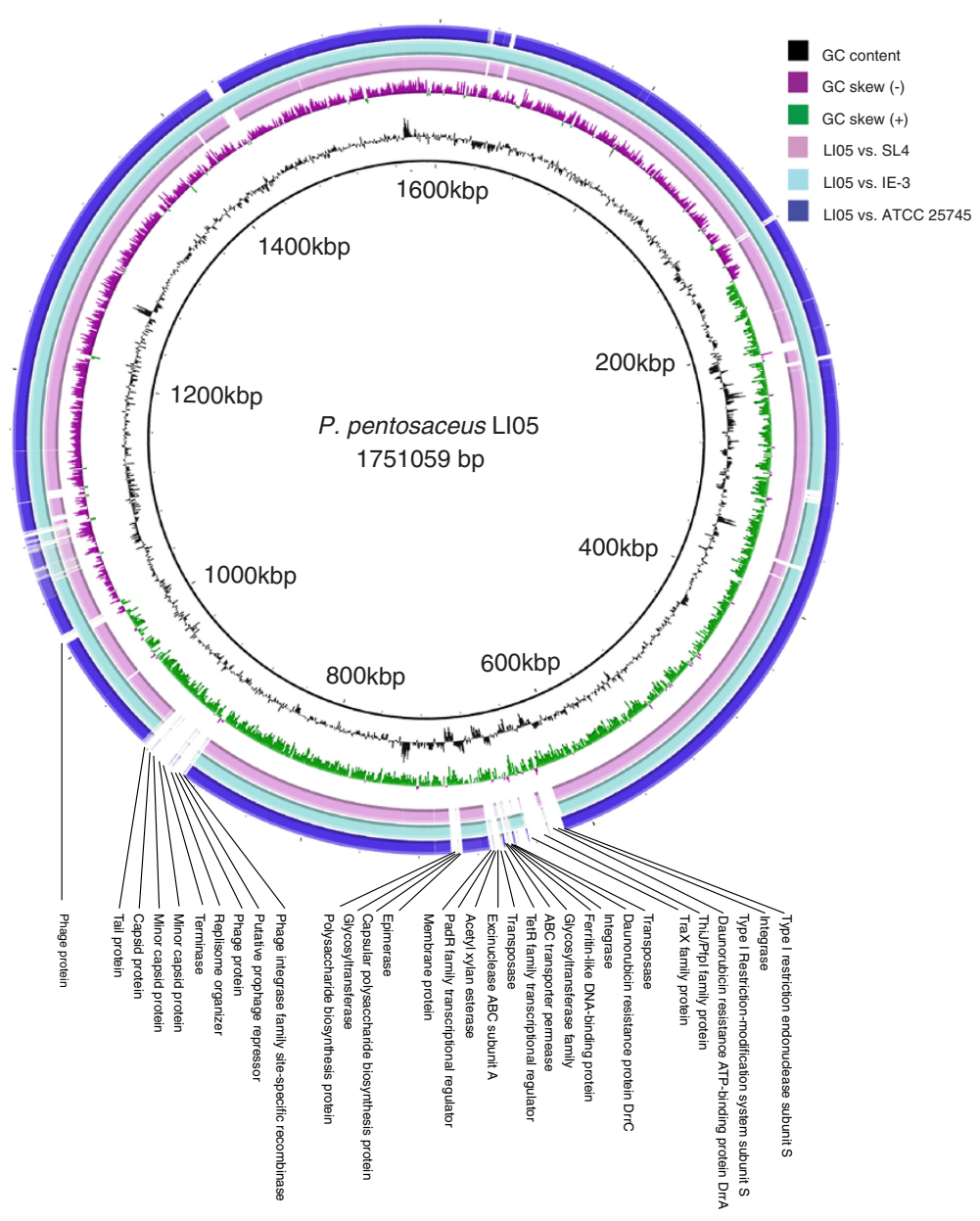

Figure 3 BRIG BLAST analysis of the $P$. pentosaceus genomes using the genome of strain LI05 as the reference. The strains and figure colors (from the center to the outside) represent LI05 vs. SL4 (pink), LI05 vs. IE-3 (light blue) and LI05 vs. ATCC 25745 (dark blue). 
Table 2 Comparison of important genes encoding stress resistance proteins in $P$. pentosaceus LI05, P. pentosaceus ATCC 25745, $P$. pentosaceus SL4 and $P$. pentosaceus IE-3

\begin{tabular}{|c|c|c|c|c|c|c|c|}
\hline \multirow[t]{2}{*}{ Characterization } & \multirow[t]{2}{*}{ Locus_tag } & \multirow[t]{2}{*}{ Size (bp) } & \multirow[t]{2}{*}{ Product description } & \multicolumn{3}{|c|}{ Identity (BLASTx) } & \multirow{2}{*}{$\begin{array}{l}\text { Max. identity } \\
\text { to proteins o } \\
\text { other species } \\
\text { (BLASTp) (\%) }\end{array}$} \\
\hline & & & & $\begin{array}{l}\text { ATCC } \\
25745 \text { (\%) }\end{array}$ & SL4 (\%) & IE-3 (\%) & \\
\hline Bile tolerance & BB06_RS0100955 & 1,026 & Choloylglycine hydrolase family protein & 99.05 & 99.25 & 100 & 69.50 \\
\hline \multirow[t]{7}{*}{ Acid tolerance } & BB06_RS0107040 & 1,518 & F0F1 ATP synthase subunit alpha & 99.74 & 99.60 & 95.39 & 92.46 \\
\hline & BB06_RS0107025 & 420 & FOF1 ATP synthase subunit epsilon & 99.76 & 99.29 & 99.76 & 84.89 \\
\hline & BB06_RS0107030 & 1,410 & FOF1 ATP synthase subunit beta & 99.86 & 99.86 & 100 & 96.16 \\
\hline & BB06_RS0107035 & 921 & F0F1 ATP synthase subunit gamma & 99.89 & 99.78 & 99.89 & 87.95 \\
\hline & BB06_RS0107050 & 522 & FOF1 ATP synthase subunit B & 99.81 & 99.81 & 100 & 90.53 \\
\hline & BB06_RS0107055 & 213 & FOF1 ATP synthase subunit C & 100 & 100 & 100 & 87.14 \\
\hline & BB06_RS0107060 & 717 & FOF1 ATP synthase subunit A & 100 & 99.86 & 99.86 & 96.22 \\
\hline \multirow[t]{6}{*}{ Universal stress resistance } & BB06_RS0103670 & 453 & Universal stress protein UspA & 99.12 & 99.34 & 99.12 & 68.42 \\
\hline & BB06_RS0106945 & 456 & Universal stress protein UspA & 100 & 100 & 100 & 90.67 \\
\hline & BB06_RS0101810 & 474 & Universal stress protein UspA & 99.37 & 99.79 & 99.79 & 70.70 \\
\hline & BB06_RS0100900 & 432 & Universal stress protein UspA & 99.54 & 99.07 & 99.77 & 99.30 \\
\hline & BB06_RS0101815 & 450 & Universal stress protein UspA & 99.33 & 99.11 & 99.11 & 93.24 \\
\hline & BB06_RS0102220 & 453 & Universal stress protein UspA & 99.78 & 99.78 & 100 & 91.21 \\
\hline \multirow{2}{*}{$\begin{array}{l}\text { Hyperosmotic and heat } \\
\text { resistance }\end{array}$} & BB06_RS0104860 & 594 & GrpE protein & 98.32 & 98.82 & 99.83 & 72.60 \\
\hline & BB06_RS0104870 & 1,131 & Molecular chaperone DnaJ & 99.03 & 98.85 & 99.82 & 88.56 \\
\hline Environmental stress resistance & BB06_RS0102555 & 1,620 & Molecular chaperone GroEL & 100 & 99.26 & 100 & 94.81 \\
\hline Oxidative stress resistance & BB06_RS0105175 & 516 & Methionine sulfoxide reductase A & 99.22 & 99.22 & 99.61 & 81.87 \\
\hline Enterocin A resistance & BB06_RS0101010 & 279 & Enterocin A Immunity family protein & 99.64 & - & 100 & 38.89 \\
\hline Arsenate resistance & BB06_RS0105725 & 354 & Arsenate reductase & 99.15 & 98.87 & 100 & 83.76 \\
\hline
\end{tabular}

Note: "-", not detected.

the genes and coding proteins related to the peptidoglycan pathway were conserved in the four strains of $P$. pentosaceus, they were not significantly similar to those of the other species. These findings will contribute to the elucidation of the mechanisms of immune regulation in $P$. pentosaceus LI05.

\section{Comparisons with other fully sequenced genomes}

Fifty-three proteins encoded by $P$. pentosaceus LI05 genes were not detected or had sequence similarities of less than $50 \%$ in the comparative analysis with the three known food-borne strains, P. pentosaceus ATCC 25745, SL4 and IE-3. Among these proteins, 21 hypothetical proteins with no clear functions were not further analyzed; the other 32 proteins are listed in Table 4, demonstrated in Figure 3, and further discussed below.

Five putative exopolysaccharide biosynthesis proteins were detected only in $P$. pentosaceus LI05, including an epimerase, a capsular polysaccharide biosynthesis protein, two glycosyltransferases (key enzymes for the biosyntheses of the exopolysaccharide repeating units) and a polysaccharide biosynthesis protein. Four of these enzymes need to be examined in further detail because they are not only potentially novel but also probably induce variations in the structures of their encoded polysaccharides that may have influenced adherence, biofilm formation and the nature of the immune response [27].

P. pentosaceus LI05 was characterized by three extraenvironmental stress tolerance proteins, including a putative ferritin-like DNA-binding protein, which maintains a steady state of iron ions and responds to stresses, such as those involving temperature, humidity, and ionizing and redox processes [28], a putative PadR family transcriptional regulator, which functions against phenolic acid stress, and a putative ThiJ/PfpI family protein, which is involved in cellular protection against environmental stresses [29].

Fourteen proteins related to the intrusion of exogenous DNA were identified in P. pentosaceus LI05. One group was comprised of twelve prophage-related proteins, including a phage integrase family site-specific recombinase, two integrases, a putative prophage repressor, two phage proteins, a replisome organizer, a terminase, two minor capsid proteins, a capsid protein and a tail protein. It is not rare for bacteria to contain multiple prophages in their chromosomes, which then constitute a 
Table 3 Comparison of important genes encoding beneficial proteins in $P$. pentosaceus LI05, $P$. pentosaceus ATCC 25745, $P$. pentosaceus SL4 and $P$. pentosaceus IE-3

\begin{tabular}{|c|c|c|c|c|c|c|c|}
\hline \multirow[t]{2}{*}{ Characterization } & \multirow[t]{2}{*}{ Locus_tag } & \multirow[t]{2}{*}{ Size (bp) } & \multirow[t]{2}{*}{ Product description } & \multicolumn{3}{|c|}{ Identity (BLASTx) } & \multirow{2}{*}{$\begin{array}{l}\text { Max. identity } \\
\text { to proteins o } \\
\text { other species } \\
\text { (BLASTp) (\%) }\end{array}$} \\
\hline & & & & $\begin{array}{l}\text { ATCC } \\
25745(\%)\end{array}$ & SL4 (\%) & IE-3 (\%) & \\
\hline \multirow[t]{6}{*}{ Adhesion } & BB06_RS0106620 & 306 & Competence protein ComGC & 99.67 & 99.67 & 100 & 76.47 \\
\hline & BB06_RS0106150 & 1,188 & Elongation factor Tu & 99.92 & 99.92 & 100 & 95.69 \\
\hline & BB06_RS0102755 & 1,323 & Enolase & 99.92 & 99.85 & 100 & 91.82 \\
\hline & BB06_RS0107170 & 330 & Pilus biosynthesis protein $\mathrm{HicB}$ & 100 & 100 & 100 & 60.91 \\
\hline & BB06_RS0108295 & 660 & Sortase & 99.70 & 99.24 & 99.85 & 76.71 \\
\hline & BB06_RS0102750 & 756 & Triosephosphate isomerase & 100 & 99.47 & 100 & 93.23 \\
\hline \multirow[t]{3}{*}{ Antimicrobial } & BB06_RS0101015 & 182 & Prebacteriocin & 100 & - & - & 96.30 \\
\hline & BB06_RS0105320 & & Lysin & 92.84 & 91.02 & - & 86.70 \\
\hline & BB06_RS0100880 & 528 & Colicin $\vee$ production family protein & 99.24 & 99.24 & 99.81 & 61.64 \\
\hline \multirow[t]{4}{*}{$\begin{array}{l}\text { Biosynthesis of } \\
\text { peptidoglycans }\end{array}$} & BB06_RS0102815 & 903 & $\begin{array}{l}\text { UDP-N-acetylenolpyruvoylglucosamine } \\
\text { reductase }\end{array}$ & 100 & 98.9 & 100 & 84.62 \\
\hline & BB06_RS0106375 & 1,368 & $\begin{array}{l}\text { UDP-N-acetylmuramoylalanine-D-glutamate } \\
\text { ligase }\end{array}$ & 99.05 & 98.90 & 100 & 74.23 \\
\hline & BB06_RS0107220 & 687 & Peptidoglycan-binding protein LysM & 99.85 & 99.71 & 99.85 & 67.54 \\
\hline & BB06_RS0100815 & 1,338 & Peptidoglycan-binding protein & 88.20 & 89.95 & 99.85 & 55.53 \\
\hline \multirow[t]{5}{*}{ Riboflavin synthesis } & BB06_RS0100520 & 471 & Riboflavin synthase, beta subunit & 98.09 & 97.74 & 60.30 & 61.43 \\
\hline & BB06_RS0100530 & 606 & Riboflavin synthase, alpha subunit & 98.84 & 97.85 & 100 & 61.14 \\
\hline & BB06_RS0100535 & 1,083 & Riboflavin biosynthesis protein RibD & 99.26 & 99.26 & 100 & 57.61 \\
\hline & BB06_RS0104850 & 945 & Riboflavin biosynthesis protein RibF & 97.46 & 99.26 & 100 & 74.84 \\
\hline & BB06_RS0105860 & 360 & $\begin{array}{l}\text { Riboflavin biosynthesis acetyltransferase } \\
\text { (GNAT) family }\end{array}$ & 100 & 99.72 & 100 & 84.14 \\
\hline \multirow[t]{2}{*}{ Folate } & BB06_RS0106895 & 1,275 & Folylpolyglutamate synthase & 99.45 & 99.53 & - & 59.52 \\
\hline & BB06_RS0105770 & 486 & Dihydrofolate reductase & 98.56 & 98.56 & 55.76 & 53.13 \\
\hline Gamma-aminobutyrate & BB06_RS0107660 & 1,452 & Gamma-aminobutyrate permease & 99.38 & 99.66 & 100 & 85.45 \\
\hline Biotin & BB06_RS0108625 & 560 & Biotin biosynthesis protein BioY & 99.47 & 99.47 & 100 & 55.19 \\
\hline \multirow[t]{2}{*}{ Thiamine } & BB06_RS0106910 & 1,188 & Thiamine biosynthesis protein Thil & 99.67 & 99.67 & 100 & 88.64 \\
\hline & BB06_RS0107185 & 942 & Thiamine biosynthesis protein ApbE & 98.09 & 98.93 & 99.79 & 63.96 \\
\hline
\end{tabular}

Note: "-", no detection.

sizable proportion of their total chromosomal material [30]. Pathogenic, commensal, and symbiotic bacteria have been observed to play roles in a variety of bacterial adaptations in hosts [31]. Phage-related proteins were encoded by genes in each of the three food-borne strains. The other genes detected in the P. pentosaceus LI05 included two encoding bacterial DNA type I restriction endonucleases, which are involved in prokaryotic DNA restrictionmodification mechanisms that protect the bacteria against invading foreign DNA [32].

Two putative doxorubicin-daunorubicin resistance proteins existed in $P$. pentosaceus LI05. One ORF encoded DrrA, which is part of the $A B C$ transporter complex DrrAB. The other ORF encoded DrrC, which is part of the $\mathrm{ABC}$ transporter permease protein. This finding partially reflects the complex interactions between drugs and gut-associated microbes [33]. Both daunorubicin and doxorubicin are antitumor drugs and are thus not suitable for antibacterial applications. Therefore, these two genes will not affect the control of $P$. pentosaceus LI05.

Additionally, there were eight extra putative multifunctional proteins in P. pentosaceus LI05. These included a TetR family transcriptional regulator, an $\mathrm{ABC}$ transporter permease, an exonuclease $\mathrm{ABC}$ subunit $\mathrm{A}$, a transposase, an acetyl xylan esterase, a PadR family transcriptional regulator, a membrane protein and a TraX family protein.

\section{Conclusions}

Strains of $P$. pentosaceus are frequently identified in food and in the human gastrointestinal tract and are known to reduce inflammation, encephalopathy, obesity and fatty liver in animals. Therefore, it is imperative to study 
Table 4 Genes and their encoded proteins detected in P. pentosaceus LI05 with sequence similarities of less than $50 \%$ with sequences from both $P$. pentosaceus ATCC 25745 and $P$. pentosaceus SL4

\begin{tabular}{|c|c|c|c|c|c|c|}
\hline \multirow[t]{2}{*}{ Locus } & \multirow{2}{*}{$\begin{array}{l}\text { Size } \\
\text { (bp) }\end{array}$} & \multirow{2}{*}{$\begin{array}{l}\text { Predicted function } \\
\text { By BLASTp }\end{array}$} & \multicolumn{2}{|l|}{ Best BLASTp hit } & \multirow{2}{*}{$\begin{array}{l}\% \text { Query } \\
\text { cover }\end{array}$} & \multirow{2}{*}{$\begin{array}{l}\% \text { Amino } \\
\text { acid identity }\end{array}$} \\
\hline & & & Accession no. & Organism & & \\
\hline BB06_RS0102945 & 1,110 & Type I restriction endonuclease subunit S & WP_000072560.1 & Staphylococcus aureus & 100 & 49.34 \\
\hline BB06_RS0102950 & 912 & Integrase & WP_006845852.1 & Weissella koreensis & 100 & 74.26 \\
\hline BB06_RS0102955 & 672 & $\begin{array}{l}\text { Type I restriction-modification system, } \\
\text { specificity subunit S }\end{array}$ & WP_003595917.1 & Lactobacillus casei & 100 & 54.71 \\
\hline BB06_RS0102980 & 936 & $\begin{array}{l}\text { Daunorubicin resistance ATP-binding } \\
\text { protein DrrA }\end{array}$ & YP_004841605.1 & $\begin{array}{l}\text { Lactobacillus sanfranciscensis } \\
\text { TMW } 1.1304\end{array}$ & 100 & 62.06 \\
\hline BB06_RS0102995 & 678 & ThiJ/Pfpl family protein & WP_010770374.1 & Enterococcus caccae & 99 & 71.42 \\
\hline BB06_RS0103035 & 633 & TraX family protein & YP_006726711.1 & Lactobacillus buchneri CD034 & 99 & 50.90 \\
\hline BB06_RS0103095 & 618 & Transposase & YP_005004471.1 & Pediococcusclaussenii ATCC BAA-344 & 100 & 98.05 \\
\hline BB06_RS0103100 & 2,268 & Daunorubicin resistance protein DrrC & WP_003680292.1 & Lactobacillus coryniformis & 100 & 99.07 \\
\hline BB06_RS0103110 & 591 & Integrase & WP_004906016.1 & Leuconostoc citreum & 99 & 97.96 \\
\hline BB06_RS0103120 & 468 & Ferritin-like DNA-binding protein & BAN08201.1 & Lactobacillus plantarum 2025 & 100 & 99.35 \\
\hline BB06_RS0103125 & 744 & Glycosyltransferase family 2 & WP_027822873.1 & Lactobacillus plantarum & 100 & 99.60 \\
\hline BB06_RS0103130 & 1,248 & $\mathrm{ABC}$ transporter permease & WP_027822874.1 & Lactobacillus plantarum & 100 & 99.76 \\
\hline BB06_RS0103135 & 672 & TetR family transcriptional regulator & WP_027822875.1 & Lactobacillus plantarum & 100 & 99.55 \\
\hline BB06_RS0103140 & 339 & Transposase & WP_015474731.1 & Lactobacillus brevis & 95 & 99.07 \\
\hline BB06_RS0103155 & 1,308 & Excinuclease $A B C$ subunit $A$ & WP_024862991.1 & Pediococcus acidilactici & 99 & 72.51 \\
\hline BB06_RS0103175 & 859 & Acetyl xylan esterase & WP_025478109.1 & Enterococcus saccharolyticus & 99 & 88.97 \\
\hline BB06_RS0103180 & 561 & PadR family transcriptional regulator & WP_017552090.1 & Bacillus coagulans & 100 & 99.43 \\
\hline BB06_RS0103200 & 906 & Membrane protein & 024625654.1 & Lactobacillus fabifermentans & 99 & 45.18 \\
\hline BB06_RS0103305 & 930 & Epimerase & WP_021357793.1 & Lactobacillus plantarum & 99 & 62.50 \\
\hline BB06_RS0103310 & 660 & $\begin{array}{l}\text { Capsular polysaccharide biosynthesis } \\
\text { protein }\end{array}$ & WP_003680917.1 & Lactobacillus coryniformis & 93 & 60.68 \\
\hline BB06_RS0103315 & 846 & Glycosyltransferase & WP_003638227.1 & Lactobacillus pentosus & 96 & 45.39 \\
\hline BB06_RS0103320 & 987 & Polysaccharide biosynthesis protein & YP_004889104.1 & Lactobacillus plantarum WCFS1 & 98 & 48.32 \\
\hline BB06_RS0104430 & 1,173 & $\begin{array}{l}\text { Phage integrase family site-specific } \\
\text { recombinase }\end{array}$ & WP_004165738.1 & Pediococcus acidilactici DSM 20284 & 99 & 75.19 \\
\hline BB06_RS0104450 & 398 & Putative prophage repressor & WP_007289487.1 & Thermosinus carboxydivorans & 97 & 41.98 \\
\hline BB06_RS0104515 & 768 & Phage protein & ERL43462.1 & Lactobacillus plantarum JDM1 & 64 & 48 \\
\hline BB06_RS0104520 & 692 & Replisome organizer & WP_004165758.1 & Pediococcus acidilactici & 99 & 66.09 \\
\hline BB06_RS0104600 & 1,374 & Terminase & WP_002318686.1 & Enterococcus faecium & 100 & 60.18 \\
\hline BB06_RS0104605 & 1,548 & Minor capsid protein & WP_022638369.1 & Lactobacillus plantarum & 98 & 58.75 \\
\hline BB06_RS0104610 & 1,134 & Minor capsid protein & WP_016511174.1 & Lactobacillus plantarum & 99 & 49.47 \\
\hline BB06_RS0104645 & 462 & Capsid protein & WP_002314916.1 & Enterococcus faecium & 92 & 72.03 \\
\hline BB06_RS0104660 & 5,241 & Tail protein & WP_002820753.1 & Oenococcus oeni & 73 & 35.42 \\
\hline BB06_RS0105620 & 810 & Phage protein & WP_012678830.1 & Streptococcus equi & 92 & 33.46 \\
\hline
\end{tabular}

the probiotic ability of this organism. Future studies will focus on delineating the interactions between the host and $P$. pentosaceus. The genome sequences of $P$. pentosaceus LI05 isolated from the human gastrointestinal tract allow for a deeper understanding of its probiotic abilities, facilitating the future development of drugs for microbiota-related diseases.

\section{Availability of supporting data}

The whole-genome sequencing project of $P$. pentosaceus LI05 has been submitted to GenBank under the project accession number PRJNA237570. The project version entailing the draft assembly described herein has been deposited under the accession number JDVW00000000. 


\section{Additional files}

Additional file 1: Figure S1. Scanning electron micrograph (A) and transmission electron micrograph (B) of P. pentosaceus LI05.

Additional file 2: Table S1. Ability of $P$. pentosaceus LI05 to grow using specific carbohydrates.

\section{Competing interests}

The authors declare no competing interests.

\section{Authors' contributions}

$L-J L$ designed the study, interpreted the results and edited the manuscript. $\mathrm{L}-\mathrm{XL}$ and $\mathrm{Y}-\mathrm{DL}$ conducted the Illumina sequencing, performed the assemblies, analyzed the genome, and performed the annotations. X-JH provided advice related to the outbreak and strain features, characterized the strain and maintained it in pure cultures. H-YS contributed to the microbiology of the strain and prepared high-molecular-weight DNA for the genome sequencing. All authors read and approved the manuscript prior to submission.

\section{Acknowledgments}

This study was supported by the National Basic Research Program of China (973 Program) (No. 2013CB531401) and the Key Program of the National Natural Science Foundation of China (No. 81330011).

\section{Author details}

'State Key Laboratory for Diagnosis and Treatment of Infectious Disease, The First Affiliated Hospital, Zhejiang University, Hangzhou 310003, PR China. ${ }^{2}$ Food Safety Key Lab of Zhejiang Province, Zhejiang Gongshang University, Hangzhou, PR China. ${ }^{3}$ Collaborative Innovation Center for Diagnosis and Treatment of Infectious Diseases, Hangzhou, China.

Received: 19 June 2014 Accepted: 18 August 2014

Published: 30 August 2014

\section{References}

1. Wieme A, Cleenwerck I, Van Landschoot A, Vandamme P: Pediococcus Iolii DSM 19927 T and JCM 15055 T are strains of Pediococcus acidilactici. Int $J$ Syst Evol Microbiol 2012, 62:3105-3108.

2. Leroy F, Verluyten J, De Vuyst L: Functional meat starter cultures for improved sausage fermentation. Int J Food Microbiol 2006, 106:270-285.

3. Martino ME, Maifreni M, Marino M, Bartolomeoli I, Carraro L, Fasolato L, Cardazzo B: Genotypic and phenotypic diversity of Pediococcus pentosaceus strains isolated from food matrices and characterisation of the penocin operon. Antonie Van Leeuwenhoek 2013, 103:1149-1163.

4. Bengmark S: Bio-ecological control of chronic liver disease and encephalopathy. Metab Brain Dis 2009, 24:223-236.

5. Zhao X, Higashikawa F, Noda M, Kawamura Y, Matoba Y, Kumagai T, Sugiyama M: The obesity and fatty liver are reduced by plant-derived Pediococcus pentosaceus LP28 in high fat diet-induced obese mice. PLoS One 2012, 7:e30696.

6. Varsha KK, Priya S, Devendra L, Nampoothiri KM: Control of Spoilage Fungi by Protective Lactic Acid Bacteria Displaying Probiotic Properties. Appl Biochem Biotechnol 2014, 172:3402-3413.

7. Lv LX, Hu XJ, Qian GR, Zhang H, Lu HF, Zheng BW, Jiang L, Li LJ: Administration of Lactobacillus salivarius LIO1 or Pediococcus pentosaceus LI05 improves acute liver injury induced by D-galactosamine in rats. App/ Microbiol Biotechnol 2014, 98:5619-5632.

8. Dantoft SH, Bielak EM, Seo JG, Chung MJ, Jensen PR: Complete genome sequence of Pediococcus pentosaceus strain SL4. Genome Announc 2013, 26:e01106-e01113.

9. Midha S, Ranjan M, Sharma V, Kumari A, Singh PK, Korpole S, Patil PB: Genome sequence of Pediococcus pentosaceus strain IE-3. J Bacteriol 2012, 194:4468.

10. Makarova K, Slesarev A, Wolf Y, Sorokin A, Mirkin B, Koonin E, Pavlov A, Pavlova N, Karamychev V, Polouchine N, Shakhova V, Grigoriev I, Lou Y, Rohksar D, Lucas S, Huang K, Goodstein DM, Hawkins T, Plengvidhya V, Welker D, Hughes J, Goh Y, Benson A, Baldwin K, Lee JH, Díaz-Muñiz I, Dosti B, Smeianov V, Wechter W, Barabote R, et al: Comparative genomics of the lactic acid bacteria. Proc Natl Acad Sci U S A 2006, 103:15611-15616.
11. Smibert RM, Krieg NR: Phenotypic Characterization. In Methods for General and Molecular Bacteriology. Washington, DC, USA: American Society for Microbiology Press; 1994:607-654

12. Tamura K, Dudley J, Nei M, Kumar S: MEGA4: Molecular Evolutionary Genetics Analysis (MEGA) software version 4.0. Mol Biol Evol 2007, 24:1596-1599.

13. Luo R, Liu BH, Xie YL: SOAPdenovo2: an empirically improved memory-efficient short-read de novoassembler. GigaScience 2012, 1:18.

14. Delcher AL, Bratke KA, Powers EC, Salzberg SL: Identifying bacterial genes and endosymbiont DNA with Glimmer. Bioinformatics 2007, 23:673-679.

15. Lowe TM, Eddy SR: tRNAscan-SE: a program for improved detection of transfer RNAgenes in genomic sequence. Nucleic Acids Res 1997, 25:955-964.

16. Benson G: Tandem repeats finder: a program to analyze DNA sequences. Nucleic Acids Res 1999, 27:573-580.

17. Petersen TN, Brunak S, von Heijne G, Nielsen H: SignalP 4.0: discriminating signal peptides from transmembrane regions. Nat Methods 2011, 8:785-786.

18. Krogh A, Larsson B, von Heijne G, Sonnhammer EL: Predicting transmembrane protein topology with a hidden Markov model: application to complete genomes. J Mol Biol 2001, 305:567-580.

19. Markowitz VM, Chen IM, Palaniappan K, Chu K, Szeto E, Pillay M, Ratner A, Huang J, Woyke T, Huntemann M, Anderson I, Billis K, Varghese N, Mavromatis K, Pati A, Ivanova NN, Kyrpides NC: IMG 4 version of the integrated microbial genomes comparative analysis system. Nucleic Acids Res 2014, 42:D560-D567.

20. Alikhan NF, Petty NK, Ben Zakour NL, Beatson SA: BLAST Ring Image Generator (BRIG): simple prokaryote genome comparisons. BMC Genomics 2011, 12:402.

21. Senan S, Prajapati JB, Joshi CG: Whole-genome based validation of the adaptive properties of Indian origin probiotic Lactobacillus helveticus MTCC 5463. J Sci Food Agric 2014, doi:10.1002/jsfa.6721.

22. Grover S, Rashmi HM, Srivastava AK, Batish VK: Probiotics for human health -new innovations and emerging trends. Gut Pathog 2012, 4:15.

23. Kim YC, Tarr AW, Penfold CN: Colicin import into E. coli cells: a mode system for insights into the import mechanisms of bacteriocins. Biochim Biophys Acta 1843, 2014:1717-1731.

24. Pastagia M, Schuch R, Fischetti VA, Huang DB: Lysins: the arrival of pathogen-directed anti-infectives. J Med Microbio/ 2013, 62:1506-1516

25. Capozzi V, Russo P, Duenas MT, Lopez P, Spano G: Lactic acid bacteria producing B-group vitamins: a great potential for functional cereals products. Appl Microbiol Biotechnol 2012, 96:1383-1394.

26. Kanmani P, Satish Kumar R, Yuvaraj N, Paari KA, Pattukumar V, Arul V Probiotics and its functionally valuable products-a review. Crit Rev Food Sci Nutr 2013, 53:641-658.

27. Nadkarni MA, Chen Z, Wilkins MR, Hunter N: Comparative genome analysis of Lactobacillus rhamnosus clinical isolates from initial stages of dental pulp infection: identification of a new exopolysaccharide cluster. PLOS One 2014, 9:e90643.

28. Smith JL: The physiological role of ferritin-like compounds in bacteria. Crit Rev Microbiol 2004, 30:173-185.

29. Zhan D, Han W, Feng Y: Experimental and computational studies indicate the mutation of Glu12 to increase the thermostability of oligomeric protease from Pyrococcus horikoshii. J Mol Model 2011, 17:1241-1249.

30. Canchaya C, Proux C, Fournous G, Bruttin A, Brussow H: Prophage genomics. Microbiol Mol Biol Rev 2003, 67:238-276.

31. Hooper LV, Gordon I: Commensal host-bacterial relationships in the gut Science 2001, 292:1115-1118.

32. Loenen WA, Raleigh EA: The other face of restriction: modification-dependent enzymes. Nucleic Acids Res 2014, 42:56-69.

33. Saad R, Rizkallah MR, Aziz RK: Gut Pharmacomicrobiomics: the tip of an iceberg of complex interactions between drugs and gut-associated microbes. Gut Pathog 2012, 4:16.

\section{doi:10.1186/s13099-014-0036-y}

Cite this article as: LV et al:: Whole-genome sequence assembly of Pediococcus pentosaceus LI05 (CGMCC 7049) from the human gastrointestinal tract and comparative analysis with representative sequences from three food-borne strains. Gut Pathogens 2014 6:36. 